\title{
The clinical profile of patients with anorexia nervosa in Singapore: a follow-up descriptive study
}

\author{
Angeline $\underline{K u e k}^{1}$, BSc(Hons), Ranjani Utpala ${ }^{2}$, DClinPsych, Huei Yen $\underline{L e e}^{1}$, MBBCh, MMed
}

INTRODUCTION The prevalence rate of anorexia nervosa is lower in Asia than in the West, although studies have found that it is on the rise in Asia. This study aims to present the clinical profile of patients presenting with anorexia nervosa in Singapore.

METHODS The present study used archival data from the Eating Disorder Programme registry of the Department of Psychiatry, Singapore General Hospital, Singapore. Patient records from 2003 to 2010 were collected and analysed. Presenting characteristics of the patients were also compared with those of another local study conducted eight years earlier.

RESULTS From 2003 to 2010, a total of 271 patients were diagnosed with anorexia nervosa by a psychiatrist in our hospital. Of these, 251 (92.6\%) were female and 238 (87.8\%) were Chinese. Our patients had a lower mean weight (36.83 kg, $\mathrm{p}<0.001)$ and a lower mean body mass index (BMI) $\left(14.43 \mathrm{~kg} / \mathrm{m}^{2}, \mathrm{p}<0.001\right)$ than patients from the previous local study. Almost half of all our patients $(n=135,49.8 \%)$ were diagnosed with at least one psychiatric comorbidity and 50 ( $18.5 \%)$ had a history of self-harm.

CONCLUSION The presenting characteristics of our study cohort were similar to those of the Western population. However, the lower presenting weight and BMI in our cohort indicates that cases seen today are more severe than those seen eight years ago. Therefore, it is important to put in place prevention programmes to help adolescents cultivate a healthy body image as well as early intervention programmes to improve detection rates and treatment outcomes.

Keywords: anorexia nervosa, Singapore

\section{INTRODUCTION}

Anorexia nervosa (AN) is the third most common chronic illness in female adolescents. ${ }^{(1,2)}$ It was previously misconstrued to be a culture-bound disorder due to its higher prevalence rates in Western societies. ${ }^{(3,4)}$ AN negatively affects an individual's physical and mental well-being and lowers quality of life. ${ }^{(5,6)} \mathrm{A}$ review of epidemiological studies on AN from the United States and Western Europe has reported an average prevalence rate of $0.3 \%,{ }^{(4)}$ while other studies have also reported a rising incidence of eating disorders. ${ }^{(4,7)}$ This is supported by the increasing number of reports on eating disorders occurring in Asia, a region where eating disorders were previously thought to be rare. ${ }^{(8)}$

Although the prevalence rate of eating disorders in Asia is still lower than that in the West, ${ }^{(8,9)}$ studies have found that Southeast Asian individuals exhibit lower levels of body satisfaction, ${ }^{(10)}$ greater eating disorder psychopathology and greater concerns about their weight as compared to their Western counterparts. ${ }^{(11,12)}$ Kok and Tian reported that Singaporean adolescents exhibited greater levels of body dissatisfaction and a higher drive for thinness as compared to American undergraduate students. ${ }^{(13)}$ In a study involving over 200 youths living in Singapore, Wang et al reported that only $36 \%$ of the female youths surveyed were satisfied with their present weight. The authors also found that a majority of Chinese Singaporean female youths preferred thinness as an ideal body size. ${ }^{(14)}$ This is particularly worrying, as body dissatisfaction is a known risk factor for eating disorders. ${ }^{(15)}$ In fact, a Singapore study that surveyed female persons aged $12-26$ years found that $7.4 \%$ of them were at risk of developing an eating disorder. ${ }^{(16)}$

Given the evident preoccupation with weight and shape among Singaporean youth, it is not surprising that there has been an increase in the incidence of eating disorders in Singapore over the years. Ung et al reported that the number of ward admissions for eating disorders increased from $1.1 \%$ to $2.0 \%$ over a period of four years. ${ }^{(3)}$ Lee et al found that the number of patients who presented with AN in a Singapore hospital increased fourfold from 1994 to 2002, similar to that seen in the West. In the same study, the patients also presented with clinical characteristics similar to those reported in the Western literature, although Malays were underrepresented in the study. ${ }^{(17)}$

Since the publication of Lee et al's study in 2005, there have been no other studies conducted to determine the profile of patients with AN in Singapore. The aim of the present study was to address this gap in the literature. We examined the clinical profile of patients diagnosed with $\mathrm{AN}$ who were seen at a local public hospital in Singapore between 2003 and 2010. We also explored whether the differences in AN presentation among the ethnic groups, which were observed in a study by Lee et al in $2005,{ }^{(17)}$ were also present in our study.

\section{METHODS}

We conducted a review of the Eating Disorder Programme registry of the Department of Psychiatry, Singapore General Hospital, Singapore. Patient records from 1 January 2003 to 
31 December 2010 were retrieved and analysed. During the eight-year period, a total of 271 patients were diagnosed by a psychiatrist with either AN-restricting or AN-binge/purge subtype, according to the Diagnostic and Statistical Manual of Mental Disorders, fourth edition (DSM-IV) criteria. ${ }^{(18)}$ Approval was obtained from the Ethics Committee to obtain, read and analyse these patient records.

The following information was obtained from the 271 case records: gender; ethnicity; age of onset (i.e. age when characteristics of AN became apparent); age of presentation (i.e. age when the patient was first seen by the psychiatrist); presenting weight; presenting height; presenting body mass index (BMI); medical comorbidities; psychiatric comorbidities; source of referral; number of patients who were first admitted as an inpatient; number of patients who participated in the Trim and Fit (TAF) programme, a weight management programme conducted in local schools; and common triggers.

Data analysis was performed using the Statistical Package for the Social Sciences version 17.0 for Windows XP (SPSS Inc, Chicago, IL, USA). One-sample $t$-test was used to compare the presenting characteristics of the patients in the present study with those of patients from Lee et al's study. ${ }^{(17)}$ Statistical significance was set at $p<0.05$.

\section{RESULTS}

Among the 271 patients with $\mathrm{AN}, 199$ (73.4\%) patients met the criteria for $\mathrm{AN}$-restricting subtype, while the remaining patients were categorised into the AN-binge/purge subtype. Most of the patients were single $(n=263,97.0 \%)$, female $(n=251,92.6 \%)$ and Chinese $(n=238,87.8 \%)$. Patients of Malay, Indian and other ethnicities made up only $12.2 \%(n=33)$ of the patient population.

The majority of referrals came from tertiary sources ( $n=148,54.6 \%$ ), with self-referrals (i.e. self and/or parents) being the next biggest source $(n=66,24.4 \%)$. The remaining referrals came from primary care providers (i.e. private GP clinics or polyclinics). Nearly $50 \%(n=134)$ of the cases were brought to medical attention by parents who suspected that their child had an eating disorder. Among the remaining patients, 113 (41.7\%) identified the symptoms of an eating disorder themselves, while $24(8.9 \%)$ were suspected to have an eating disorder by a doctor, teacher, friend or partner. The presenting characteristics of the 271 patients are shown in Table I.

In order to examine whether there were changes in the characteristics of our study cohort as compared to those of patients from Lee et al's study, ${ }^{(17)}$ a one-sample $t$-test was performed. Significant differences between the two groups of patients were found for age of onset, presenting weight and presenting BMI. No significant difference was found for presenting age and presenting height. The patients in the present study had a significantly later mean age of onset than the patients in Lee et al's study (16.34 years vs. 15.50 years; $\mathrm{t}[270]=3.43, \mathrm{p}<0.001$, $\mathrm{d}=0.21$ ). In addition, our patients were 0.84 years older than those in Lee et al's study (95\% confidence interval [CI] 0.36-1.32). The mean duration of untreated illness in the present cohort was shorter than that in Lee et al's cohort (1.39 years vs. 2.17 years;
Table I. Characteristics of the patients with anorexia nervosa $(n=271)$.

\begin{tabular}{lc}
\hline Variable & Mean \pm SD (range) \\
\hline Presenting age $(\mathrm{yr})$ & $17.51 \pm 4.75(12-37)$ \\
Age of onset $(\mathrm{yr})$ & $16.34 \pm 4.01(8-36)$ \\
Duration of untreated illness $(\mathrm{yr})$ & $1.39 \pm 1.99(0-12)$ \\
Presenting weight $(\mathrm{kg})$ & $36.83 \pm 5.93(23.10-56.40)$ \\
Presenting height $(\mathrm{m})$ & $1.60 \pm 0.07(1.42-1.85)$ \\
Presenting BMI $\left(\mathrm{kg} / \mathrm{m}^{2}\right)$ & $14.43 \pm 1.77(9.67-18.00)$ \\
\hline
\end{tabular}

BMI: body mass index; SD: standard deviation

Table II. Frequency of psychiatric comorbidities among patients with anorexia nervosa $(n=271)$.

\begin{tabular}{lc}
\hline Variable & No. (\%) \\
\hline Psychiatric comorbidity absent & $136(50.2)$ \\
Psychiatric comorbidity present & $135(49.8)$ \\
Mood disorders & $88(32.5)$ \\
Obsessive compulsive disorder & $7(2.6)$ \\
Anxiety disorders & $1(0.4)$ \\
Substance abuse & $4(1.5)$ \\
Others & $4(1.5)$ \\
$>1$ psychiatric comorbidity & $31(11.4)$ \\
\hline
\end{tabular}

$t[270]=-6.41, p<0.001, d=-0.34)$. Patients in the present study sought treatment 0.78 years earlier than those from Lee et al's study $(95 \% \mathrm{Cl}-1.01$ to -0.54$)$.

Our patients had a significantly lower mean presenting weight than Lee et al's patients (36.83 kg vs. $39.50 \mathrm{~kg}$; $\mathrm{t}[270]=-7.42$, $\mathrm{p}<0.001, \mathrm{~d}=-0.36)$; the mean presenting weight of our patients was $2.67 \mathrm{~kg}$ lighter $(95 \% \mathrm{Cl}-3.38$ to -1.96$)$. Our patients also had a significantly lower presenting BMI than those from Lee et al's study $\left(14.43 \mathrm{~kg} / \mathrm{m}^{2}\right.$ vs. $15.56 \mathrm{~kg} / \mathrm{m}^{2} ; \mathrm{t}[270]=-10.49, \mathrm{p}<0.001$, $\mathrm{d}=-0.49)$; our patients' BMI was $1.13 \mathrm{~kg} / \mathrm{m}^{2}$ lower $(95 \% \mathrm{Cl}-1.34$ to -0.92$)$.

In the present study, 136 (50.2\%) patients did not have any psychiatric comorbidities, while 135 (49.8\%) did (Table II). A total of 31 patients were diagnosed with more than one psychiatric comorbidity; of these, $19(61.3 \%)$ had both a mood disorder and obsessive compulsive disorder (OCD), 11 (35.5\%) had a mood disorder and one or more other psychiatric comorbidities (e.g. anxiety, OCD, personality disorder, conduct disorder, and alcohol or substance abuse) and 1 (3.2\%) had OCD and alcohol/substance abuse. A history of self-harm and/or attempted suicide was noted in $18.5 \%$ (50/271) of patients.

In terms of medical comorbidities, 77 of the $271(28.4 \%)$ patients suffered from an eating disorder-related medical complication. Bradycardia, osteoporosis/osteopenia and hypokalaemia were the three most common comorbid medical complications. $230(84.9 \%)$ patients identified a trigger for their eating disorder. The most common triggers listed were teasing/comments by others ( $\mathrm{n}=86,31.7 \%$ ), school or work stress $(n=67,24.7 \%$ ) and dieting $(n=40,14.8 \%)$. Other commonly reported triggers included peer pressure, shape/weight concern, health reasons, family problems, boyfriend/girlfriend relationship problems, marathon training and enlistment in the 
army. Interestingly, although only 21 (7.7\%) patients identified being overweight as a trigger for their eating disorder, 28 (10.3\%) had participated in the TAF programme prior to the onset of the illness. Among those who identified a trigger, teasing/comments by others ( $n=80 / 211,37.9 \%$ ) was the main trigger identified by female patients, whereas being overweight $(n=9 / 19,47.4 \%)$ was the main trigger identified by male patients.

In terms of inpatient versus outpatient presentation, 90 (33.2\%) patients presented with $\mathrm{AN}$ as inpatients. These 90 patients had a significantly lower mean weight than those who presented with AN as outpatients $(34.91 \pm 5.78 \mathrm{~kg}$ vs. $37.80 \pm 5.78 \mathrm{~kg}$; t[269] = -3.89, $\mathrm{p}<0.001)$, as well as a lower mean BMI $\left(13.69 \pm 1.74 \mathrm{~kg} / \mathrm{m}^{2} \mathrm{vs}\right.$. $\left.14.81 \pm 1.67 \mathrm{~kg} / \mathrm{m}^{2} ; \mathrm{t}[269]=-5.11, \mathrm{p}<0.001\right)$.

We also compared the presenting age, weight, height and BMI of patients who first noticed their own eating disorder with those whose eating disorder was first noticed by another party. The latter group presented at a significantly younger mean age than those who noticed their own eating disorder $(16.86 \pm 3.96$ years vs. $18.45 \pm 5.58$ years; $\mathrm{t}[191.59]=12.07, \mathrm{p}<0.01)$. There were no significant differences in the presenting weight, height and BMI.

In the Department of Psychiatry at Singapore General Hospital, the number of new AN cases seen increased from 19 in 2003 to 41 in 2010. However, when these numbers were compared with the number of new cases seen in the same department during the time period, a slight increase in percentage of AN cases was seen only between 2006 and 2008 (Table III).

\section{DISCUSSION}

The profile of the patients in the present study were similar to that of various studies conducted in the West, in that the patients were predominantly female and in their teens. ${ }^{(19,20)}$ As Singapore is a multiethnic society comprising $74.1 \%$ Chinese, $13.4 \%$ Malays, $9.2 \%$ Indians and $3.3 \%$ other ethnicities, ${ }^{(21)}$ the ethnic composition of our study cohort is of particular interest. In the present study, the Malay and Indian populations were underrepresented $3.0 \%$ and $2.2 \%$, respectively), while 'other ethnicities' (i.e. not including Chinese, Malay and Indian) were overrepresented (7.0\%). Among the patients who were categorised as 'other ethnicities', nearly half were Caucasians. One possible explanation for this is the high levels of body image dissatisfaction, weight and shape concerns, as well as a high prevalence of eating disorders, that have been documented among Caucasian adolescents. ${ }^{(22)}$ Another possible explanation is that there is less stigma associated with seeing a psychiatrist in Western cultures as compared to Asian cultures; as a result, these individuals may have been more willing to seek treatment. ${ }^{(23)}$

It is interesting to note that Malays were underrepresented in the present study as opposed to a previous study of over 4,000 Singaporean females, which found that $20.6 \%$ of Malay girls and $9.4 \%$ of Indian girls were at risk of developing an eating disorder. ${ }^{(16)}$ Underrepresentation of Malays and Indians was also seen in Lee et al's study, although it was less pronounced. ${ }^{(17)}$ While it has been previously suggested that Malay and Indian cultures tend to place less emphasis on the thin ideal, thus accounting for the underrepresentation of $\mathrm{AN}$ in clinical settings, ${ }^{(17)}$ this
Table III. Total number of new cases seen in the Department of Psychiatry at Singapore General Hospital, Singapore, compared to number of new AN cases seen between 2003 and 2010.

\begin{tabular}{lcc}
\hline Year & No. of new cases & No. of new cases of AN (\%) \\
\hline 2003 & 694 & $19(2.74)$ \\
2004 & 1,233 & $18(1.46)$ \\
2005 & 1,393 & $19(1.36)$ \\
2006 & 1,481 & $42(2.84)$ \\
2007 & 1,676 & $49(2.92)$ \\
2008 & 1,429 & $47(3.29)$ \\
2009 & 1,656 & $36(2.17)$ \\
2010 & 1,854 & $41(2.21)$ \\
\hline
\end{tabular}

AN: anorexia nervosa

explanation is based on anecdotal rather than empirical evidence. Although it is possible that there may be cultural factors inherent in these two groups that serve as protective factors, we cannot rule out the possibility that the underrepresentation may also be due to the Malay population in Singapore being less likely to seek help for psychiatric illnesses. ${ }^{(24,25)}$

While there is no current literature regarding the mental health help-seeking behaviour of Singaporean Indians, there is evidence that Indian women living in the United Kingdom are less likely to seek help for mental illness as compared to Caucasian women residents, due to a lack of knowledge about the illness and the belief that treatment would be less beneficial. ${ }^{(26)}$ The aforementioned reasons could be extrapolated to the Singaporean Indian population, and this cultural factor might be compounded by the poor eating disorder literacy in Singapore. ${ }^{(27)}$ Hence, education and outreach programmes targeted at the Malay and Indian communities in Singapore should be increased to ensure that these groups seek help for mental health problems such as eating disorders.

In the Department of Psychiatry at Singapore General Hospital, the ratio of new $\mathrm{AN}$ cases and the total number of new cases seen each year remained relatively stable. Although the percentage of new AN cases seen each year did not increase significantly, it should be noted that staffing capacity could have played a role in the modest increase. The number of doctors seeing new general psychiatry cases increased from 2.5 full-time equivalent (FTE) to approximately 6 FTE between 2003 and 2010 (140\% increase), while the number of doctors seeing new eating disorder cases increased from 0.5 FTE to 1 FTE (100\% increase). It should also be noted that, while the percentage of new general psychiatry cases increased by about $167 \%$ in 2003-2010, the percentage of new AN cases also increased by about $115 \%$, which is of great concern. This increase in the number of individuals seeking treatment for $\mathrm{AN}$ could have been due to the establishment of a specialist eating disorders treatment programme in the hospital in 2003 and the organisation of several campaigns by the hospital to raise awareness of eating disorders (e.g. the annual eating disorder week).

It is important to note that although the duration of untreated illness in the present study was lower than that reported by Lee et $\mathrm{al}^{(17)}$ our patients had significantly lower weights and BMIs 
at presentation. This indicates that the recent cases of $\mathrm{AN}$ seen in the local population were more severe than those seen eight years ago, even though people are more aware of eating disorders and seeking treatment earlier. This increase in severity may be due to today's youth being more exposed to digital and online media (where the thin ideal is glorified) at a younger age. ${ }^{(28)}$ The link between media use and eating disorders has been extensively researched, with evidence suggesting that pressure from the media can increase the risk of an individual developing an eating disorder. ${ }^{(29)}$ A study by Lin et al found that $45 \%$ of the Singaporean youth surveyed $(n=401)$ used the Internet at least five days a week and although the average age of these respondents was 14.2 years, most had already been using the computer for the past six years. ${ }^{(30)}$ There is also evidence that Singaporean parents tend to underestimate their child's engagement in risky Internet behaviours and that adolescents tend to have unmonitored Internet use. ${ }^{(31)}$ It was reported that patients with eating disorders tend to use pro-eating disorder websites to obtain tips and advice, and adolescents exposed to these websites are more likely to have higher levels of body dissatisfaction, decreased quality of life and longer durations of eating disorders. ${ }^{(32)}$ Hence, with earlier and increased exposure to digital and online media as well as networking sites, youth today are not only more inundated by the notion that thin is beautiful but also have ready access to information on weight loss methods.

The present study saw more severe cases of AN than Lee et al's study. ${ }^{(17)}$ This could be due to the fact that AN patients tend to be resistant to treatment, ${ }^{(33)}$ i.e. patients may try to hide their eating disorder. Approximately $50 \%$ of our patients were brought for treatment by their parents. Due to the low eating disorder literacy in Singapore, it is possible that parents do not recognise signs of AN until their child is severely underweight. ${ }^{(27)}$ The poor help-seeking rates for mental health problems in Singapore ${ }^{(27)}$ could have also contributed to the increased illness severity. It is possible that even after an eating disorder is eventually noticed by family members, the patient (and family members) may still not seek treatment promptly due to misconceptions about the illness or the stigma associated with mental illness. Treatment may only be sought when the illness is very severe and requires medical attention. Therefore, there is a critical need for eating disorder literacy programmes to be set up for not only youth but also their parents and educators.

In the present study, almost half of the patients had a comorbid psychiatric diagnosis in addition to AN. Mood disorders were the most common, with over a third of the patients having this psychiatric comorbidity. These findings are consistent with those of previous studies. Ivarsson et al found that individuals diagnosed with AN were more likely to have a depressive disorder as compared to control participants. ${ }^{(34)}$ Another study reported that $22.2 \%$ of outpatients with AN engaged in self-harming behaviours and $11.3 \%$ had a history of attempted suicide. ${ }^{(35)}$ In the present study, almost $20 \%$ of the patients with AN had a history of self-harm or suicidal behaviour. Given that the mean number of suicides expected in patients with AN is eight times higher than that in the general population, ${ }^{(36)}$ and teenage patients are more emotionally vulnerable, ${ }^{(37)}$ the increase in AN severity observed in the present study is worrying.

Almost a third of the patients seen in the present study had at least one comorbid medical complication. Osteoporosis, sinus bradycardia and hypokalaemia were some of the more common complications encountered. A study conducted by Katzman reported similar medical complications in adolescents with AN. ${ }^{(38)}$ The fact that these medical complications were seen at the patient's first visit indicates the rapid progress in severity of illness. This is further supported by the fact that a third of our patients first presented as an inpatient.

The most common reason cited for the patients' desire for weight loss was comments or teasing by others. It has been shown that younger teens are more easily influenced and, thus, more susceptible to peer pressure. ${ }^{(39)}$ In Kok and Tian's survey conducted among Singaporean schoolgirls, 38\% of them admitted to being teased about being overweight; of these, $78 \%$ felt embarrassed and $69 \%$ resorted to some form of dieting as a result. ${ }^{(13)}$ Although the mean $\mathrm{BMI}$ of the schoolgirls surveyed was $19.58 \mathrm{~kg} / \mathrm{m}^{2}$ (normal BMI range according to the World Health Organization $\left.{ }^{(40)}\right), 56 \%$ of the girls felt that they were too fat, ${ }^{(13)}$ which is worrying. Given that weight-related teasing is a known risk factor for development of eating disorders, ${ }^{(41)}$ there is a need for educators and parents to be informed about the dangers of weight-related teasing so that this problem can be addressed in a constructive manner. More efforts to reduce the importance placed on weight/body size and to help young people focus on developing a healthy body image (e.g. through media literacy programmes) are also necessary.

One limitation of the present study is the retrospective nature of data collection. Thus, the quality of the information collected was subject to patient recall bias and was dependent on how well the case records were documented. Furthermore, as the intake assessments were conducted by different psychiatrists, the diagnoses recorded could have been affected by the individual clinicians' judgement and not entirely based on the objective use of a validated diagnostic tool. There might have been low inter-rater reliability, and interviewer bias was likely to have impacted the results. The generalisability of our findings is also somewhat limited by the fact that the data was collected from a single site. Finally, the profile of the patients who presented with AN in our hospital may be different from that of patients seen in other local settings.

To date, the present study is the largest Singapore study on AN conducted in a clinical population. Patients were found to have a similar clinical profile as their Western counterparts. However, they were more severely ill than patients in a previous local report, ${ }^{(17)}$ with several diagnosed with various psychiatric and medical comorbidities. This is the second study conducted in Singapore that found an underrepresentation of Indians and Malays in a clinical AN sample. It would be worthwhile for future studies to examine the cultural differences within Singapore to better understand how they affect AN development. Given the increase in AN clinical presentations, there is a need for prevention programmes to be undertaken locally in order to help 
adolescents cultivate a healthy body image. Early intervention programmes are also necessary to facilitate early detection and improve treatment outcomes. Large-scale epidemiological studies should be conducted to gather more accurate data on the prevalence rates of eating disorders in Singapore.

\section{REFERENCES}

1. Ung EK. Eating disorders in Singapore: a review. Ann Acad Med Singapore $2003 ; 32: 19-24$.

2. Herpertz-Dahlmann B. Adolescent eating disorders: definition, symptomatology, epidemiology and comorbidity. Child Adolesc Psychiatr Clin N Am 2008; 18:31-47.

3. Ung EK, Lee S, Kua EH. Anorexia nervosa and bulimia - a Singapore perspective. Singapore Med J 1997; 38:332-5.

4. Hoek HW, van Hoeken D. Review of the prevalence and incidence of eating disorders. Int J Eat Disord 2003; 34:383-96.

5. Pike KM. Long-term course of anorexia nervosa: response, relapse, remission, and recovery. Clin Psychol Rev 1998; 18:447-75.

6. Mitchison D, Hay P, Mond J, Slewa-Younan S. Self-reported history of anorexia nervosa and current quality of life: findings from a community-based study. Qual Life Res 2013; 22:273-81.

7. Micali N, Hagberg KW, Petersen I, Treasure JL. The incidence of eating disorders in the UK in 2000-2009: findings from the General Practice Research Database. BMJ Open 2013; 3:e002646.

8. Tsai G. Eating disorders in the Far East. Eat Weight Disord 2000; 5:183-97.

9. Chisuwa N, O'Dea JA. Body image and eating disorders amongst Japanese adolescents. A review of the literature. Appetite 2010; 54:5-15.

10. Kennedy MA, Templeton L, Gandhi A, Gorzalka BB. Asian body image satisfaction: ethnic and gender differences across Chinese, Indo-Asian, and European-descent students. Eat Disord 2004; 12:321-36.

11. Soh NL, Touyz S, Dobbins TA, et al. Restraint and eating concern in North European and East Asian women with and without eating disorders in Australia and Singapore. Aust N Z J Psychiatry 2007; 41:536-45.

12. Mond JM, Chen A, Kumar R. Eating-disordered behavior in Australian and Singaporean women: a comparative study. Int J Eat Disord 2010; 43:717-23.

13. Kok LP, Tian CS. Susceptibility of Singapore Chinese schoolgirls to anorexia nervosa - Part 1 (Psychological factors). Singapore Med J 1994; 35:481-5.

14. Wang MC, Ho TF, Anderson JN, Sabry ZI. Preference for thinness in Singapore, a newly industrialised society. Singapore Med J 1999; 40:502-7.

15. Grabe S, Ward LM, Hyde JS. The role of the media in body image concerns among women: a meta-analysis of experimental and correlational studies. Psychol Bull 2008; 134:460-76.

16. Ho TF, Tai BC, Lee EL, Cheng S, Liow PH. Prevalence and profile of females at risk of eating disorders in Singapore. Singapore Med J 2006; 47:499-503.

17. Lee HY, Lee EL, Pathy P, Chan YH. Anorexia nervosa in Singapore in Singapore: an eight-year retrospective study. Singapore Med J 2005; 46:275-81.

18. American Psychiatric Association. Diagnostic and Statistical Manual of Mental Disorders, 4th ed. Washington DC: American Psychiatric Press, 1994: 544-5.

19. Bulik CM, Sullivan PF, Tozzi F, et al. Prevalence, heritability, and prospective risk factors for anorexia nervosa. Arch Gen Psychiatry 2006; 63:305-12.
20. Hindler CG, Crisp AH, McGuigan S, Joughin N. Anorexia nervosa: change over time in age of onset, presentation and duration of illness. Psychol Med 1994; 24:719-29.

21. Department of Statistics, Ministry of Trade \& Industry. Census of Population 2010 Advance Census Release. Singapore: Department of Statistics, Ministry of Trade \& Industry, 2010.

22. Makino M, Tsuboi K, Dennerstein L. Prevalence of eating disorders: a comparison of Western and non-Western countries. MedGenMed 2004; 6:49.

23. Leong FT, Lau AS. Barriers to providing effective mental health services to Asian Americans. Ment Health Serv Res 2001; 3:201-14.

24. Ang AWK, Ko SM, Kua EH. Psychiatric referrals from an accident and emergency department in Singapore. J Accid Emerg Med 1995; 12:119-22.

25. Peh AL, Tay LK. Demographic profile and clinical features of patients with bipolar disorder in an outpatient setting in Singapore. Singapore Med J 2008; 49:380-3.

26. Taylor R, Brown JS, Weinman J. A comparison of the illness perceptions of North Indian and white British women. J Ment Health 2013; 22:22-32.

27. Chen A, Mond JM, Kumar R. Eating disorders mental health literacy in Singapore: beliefs of young adult women concerning treatment and outcome of bulimia nervosa. Early Interv Psychiatry 2000; 4:39-46.

28. Andrist LC. Media images, body dissatisfaction, and disordered eating in adolescent women. MCN Am J Matern Child Nurs 2003; 28:119-23.

29. Wilksch SM, Tiggemann M, Wade TD. Impact of interactive school-based media literacy lessons for reducing internalization of media ideals in young adolescent girls and boys. Int J Eat Disord 2006; 39:385-93.

30. Lin WY, Cheong PH, Kim YC, Jung JY. Becoming citizens: youths' civic uses of new media in five digital cities in East Asia. J Adolescent Res 2010; 25:839-57.

31. Liau AK, Khoo A, Ang PH. Parental awareness and monitoring of adolescent internet use. Curr Psychol 2008; 27:217-33.

32. Borzekowski DL, Schenk S, Wilson JL, Peebles R. e-Ana and e-Mia: A content analysis of pro-eating disorder Web sites. Am J Public Health 2010; 100:1526-34.

33. Connan F, Treasure J. Working with adults with anorexia nervosa in an out-patient setting. Adv Psychiatr Treat 2000; 6:135-44.

34. Ivarsson T, Rastam M, Wentz E, Gillberg IC, Gillberg C. Depressive disorders in teenage-onset anorexia nervosa: a controlled longitudinal, partly community-based study. Compr Psychiatry 2000; 41:398-403.

35. Levitt JL, Sansone RA, Cohn L, eds. Self-Harm Behaviors and Eating Disorders: Dynamics, Assessment and Treatment. New York: Brunner-Routledge, 2004.

36. Pompili M, Mancinelli I, Girardi P, Ruberto A, Tatarelli R. Suicide in Anorexia nervosa: a meta-analysis. Int J Eat Disord 2004; 36:99-103.

37. Chamberlain LB. The Amazing Teen Brain: What Every Child Advocate Needs to Know [Online]. Available at: http://www.hawaiilure.com/kiwanis/ teenbrain.pdf. Accessed September 11, 2014.

38. Katzman DK. Medical complications in adolescents with anorexia nervosa: a review of the literature. Int J Eat Disord 2005; 37:S52-9.

39. Steinberg L, Monahan KC. Age differences in resistance to peer influence. Dev Psychol 2007; 43:1531-43.

40. World Health Organization. Obesity: preventing and managing the global epidemic. Report of a WHO Consultation. WHO Technical Report Series 894. Geneva: World Health Organization, 2000.

41. Haines J, Neumark-Sztainer D, Eisenberg ME, Hannan PJ. Weight teasing and disordered eating behaviors in adolescents: longitudinal findings from Project EAT (Eating Among Teens). Pediatrics 2006; 117:e209-15. 